\title{
Absolute Cooling Rates of Freely Decaying Fullerenes
}

\author{
A. E. K. Sundén \\ Department of Physics, University of Gothenburg, 41296 Gothenburg, Sweden \\ M. Goto, J. Matsumoto, and H. Shiromaru \\ Department of Chemistry, Tokyo Metropolitan University, 1-1 Minamiosawa, Hachiouji-shi, Tokyo 192-0397, Japan \\ H. Tanuma and T. Azuma \\ Department of Physics, Tokyo Metropolitan University, 1-1 Minamiosawa, Hachiouji-shi, Tokyo 192-0397, Japan \\ J. U. Andersen \\ Institute of Physics and Astronomy, University of Aarhus, DK-8000 Aarhus C, Denmark \\ S.E. Canton \\ Chemical Physics Department, Lund University, P.O. Box 124, 22100 Lund, Sweden \\ K. Hansen \\ Department of Physics, University of Gothenburg, 41296 Gothenburg, Sweden
}

(Received 30 April 2009; published 28 September 2009)

\begin{abstract}
The cooling rates of $\mathrm{C}_{60}^{-}$have been measured in an electrostatic storage ring between several hundred $\mu \mathrm{s}$ and several tens of ms with one-photon laser excitation. The absolute energy scale is established by the photon energy, and the cooling time interval is derived from the nonexponential decay of the ensemble of hot molecules. The energy decreases due to the combined action of depletion and thermal emission of IR photons with a total energy loss rate that varies inversely proportional to time, $0.9 \mathrm{eV} / t$. The radiative component decreases from a few hundred $\mathrm{eV} / \mathrm{s}$ at submillisecond time scales to several tens of $\mathrm{eV} / \mathrm{s}$ at $20 \mathrm{~ms}$ and confirms that the crossover from depletion to predominantly radiative cooling occurs around $5 \mathrm{~ms}$. The method is applicable to any large molecule or cluster which decays spontaneously, irrespective of the specific decay channel.
\end{abstract}

DOI: 10.1103/PhysRevLett.103.143001

An important motivation for the study of clusters is their nonmonotonic approach to bulk. Both geometry and energetics can change strongly from one size to the next, as witnessed by variations in electron affinity, ionization energies, and chemical reactivities or simply mass abundances spectra. Although the existence of these size-to-size variations is very well established, measurements of absolute values of binding energies and more generally thermodynamic properties of clusters and large molecules remain a challenging task. One of the main difficulties is the determination of the excitation energy when using unimolecular processes to study, e.g., binding energies of medium size or large molecules and clusters. Important progress has been made with the measurement of heat capacities of sodium clusters [1], binding energies of gold and silver clusters [2] and heat capacities of aluminum clusters [3].

In this Letter we report on measurements of the absolute cooling rates of freely decaying fullerenes, utilizing the systematics associated with a very broad excitation energy distribution. Such experiments automatically provide the highest possible nonforced cooling rates of molecules that occur at a given time scale and are highly relevant for
PACS numbers: 31.70.Hq, 33.80.Eh, 36.40.Wa, 37.20.+j

the formation and decay of nanoparticles and their structure in nonequilibrium conditions [4-7]. Vacuum cooling rates are also directly relevant for the astrophysics of charged polyaromatic hydrocarbons present in the interstellar gas [8-10].

Wide internal energy distributions give rise to nonexponential decay rates, although the unimolecular decay of individual clusters or molecules is well described as thermally activated decay processes [11]. In the absence of radiative cooling or other competing channels, the decay rate follows a $1 / t$ profile with minor corrections from, e.g., finite heat capacities. Radiative cooling will suppress the decay strongly, but it remains nonexponential. The nonexponential decay is the key to the analysis performed in this work because it has a specific zero of time, contrary to a single exponential decay which has a characteristic time scale. Reheating the broad energy distribution ensemble with a single photon shifts it up in energy and causes an enhanced decay which can be identified with a shift backward in time.

The data were recorded at the TMU storage ring at Tokyo Metropolitan University (TMU E-ring) [12]. Reference spectra without photon absorption were also 
recorded at the storage ring ELISA in Aarhus, Denmark [13]. The rings are electrostatic and of similar design. The anions are produced hot at temperatures where they decay spontaneously by thermionic emission from the earliest times detected, a few hundred $\mu \mathrm{s}$, and onwards. In ELISA the ions are produced in a plasma source and in the TMU E-ring with a laser ablation source. No difference was found between the decay spectra recorded with the two rings, strongly suggesting that the initial excitation energy distribution does not influence the conclusions drawn. The ions were accelerated to $15 \mathrm{keV}$ before injection into the TMU E-ring where they circulated with a period of $120 \mu \mathrm{s}$. The decay rates were measured by detecting the neutral decay products with a channel plate detector located at the end of one of the straight sections. The mass selection was accomplished by pulsed field rejection of the unwanted masses during the ca. 24 revolutions completed in the first three milliseconds of ion storage [12]. At times varying between 4 and $35 \mathrm{~ms}$ a tunable optical parametrical oscillator nanosecond laser was fired and the ions irradiated with photons with energies between 1.9 and $2.7 \mathrm{eV}$. The fluence was kept low enough to guarantee that only single photon absorption contributed to the signal. Figure 1 shows two spectra after absorption of photons of different energies at time $t_{\text {las }}=12.5 \mathrm{~ms}$. Figure 2 illustrates how the enhanced signal, $I_{e}(t)$, depends on the fraction, $p$, of ions that absorb a photon, and the photon energy $h \nu$. The spontaneous decay $I(t)$ originates from decay of the distribution described by the dotted curve for the example of $t=$ $5 \mathrm{~ms}$, given by $\exp [-k(E) 5 \mathrm{~ms}]$. The laser enhanced signal originates from the fraction $p$ of the molecules that have absorbed a photon. They give a signal of $p I(t-\Delta t)$, where $\Delta t$ is a time to be fitted, plus the remaining part of the signal $(1-p) I(t)$. The total, laser enhanced signal will be

$$
I_{e}(t)=p I(t-\Delta t)+(1-p) I(t) .
$$

The value of $p$ is proportional to the photoabsorption cross section [14]. The average cooling rate between $t_{\text {las }}-\Delta t$

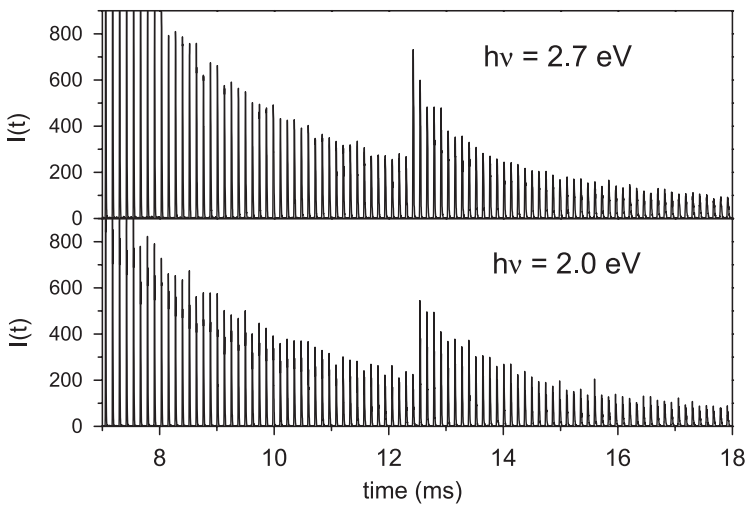

FIG. 1. Spectra for photon energies $2.7 \mathrm{eV}$ (top) and $2.0 \mathrm{eV}$ (bottom). The photon absorption probability $p$ (see below) was $4 \%$ for both spectra. and $t_{\mathrm{las}}$ is then $h \nu / \Delta t$. In practice we find $\Delta t$ by fitting $1 /\left[I_{e}(t)-I(t)\right]$ versus time. This is very close to a straight line because the decay for short times has a $1 / t$ behavior. The small difference between the ordinate intercept and the value of $t_{\text {las }}-\Delta t$ is easily included in the analysis using Eq. (1). Figure 3 illustrates the procedure with two examples.

The times $t_{\text {las }}-\Delta t$ and $t_{\text {las }}$ are very different for all the measured points, and to get quasi-instantaneous values, rates for the same laser firing times but different photon energies are subtracted, giving cooling rates for the time intervals $\left[t_{\text {las }}-\Delta t_{1}, t_{\text {las }}-\Delta t_{2}\right]$ with the photon energy differences $h \nu_{1}-h \nu_{2}$. This procedure gives cooling rates for short times. Likewise, if the backshifted times $t_{\text {las }}-\Delta t$ of two different laser firing times and photon energies are identical, the cooling rates for the time interval $\left[t_{\text {las, }, 1}, t_{\text {las }, 2}\right]$ can be calculated, yielding long time cooling rates. Selecting the time intervals that span less than a factor of 2 gives the resulting curve in Fig. 4.

The energy loss rate can be separated into the depletion part and the part due to radiative cooling by considering the deviation of the measured signal from a $1 / t$ decay. A good fit was provided by $I(t) t=a_{0} \exp \left(a_{1} t+a_{2} t^{2}\right) / \ln (\omega t)^{2}$, with $a_{1}=-122 \mathrm{~s}^{-1}, \quad a_{2}=1320 \mathrm{~s}^{-2}, \quad$ and $\omega=$ $3 \times 10^{12} \mathrm{~s}^{-1}$ between 3 and $25 \mathrm{~ms}$. Nonzero values of $a_{1}, a_{2}$ indicate the presence of radiative cooling. It is convenient to parametrize the decay rate with an Arrhenius-type formula for which the highest energy in the ensemble at time $t$ is, in the absence of cooling, given by $E_{m}=\Phi C_{v} /\left[k_{B} \ln (\omega t)\right]+E_{0} . \Phi$ is the activation energy (electron affinity), $C_{v}$ the heat capacity, $\omega$ the frequency factor for the electron emission rate constant, and $E_{0}$ includes the constant term in the caloric curve $E=$ $C T+E_{0}^{\prime}$ and the finite heat bath correction [11]. Note that

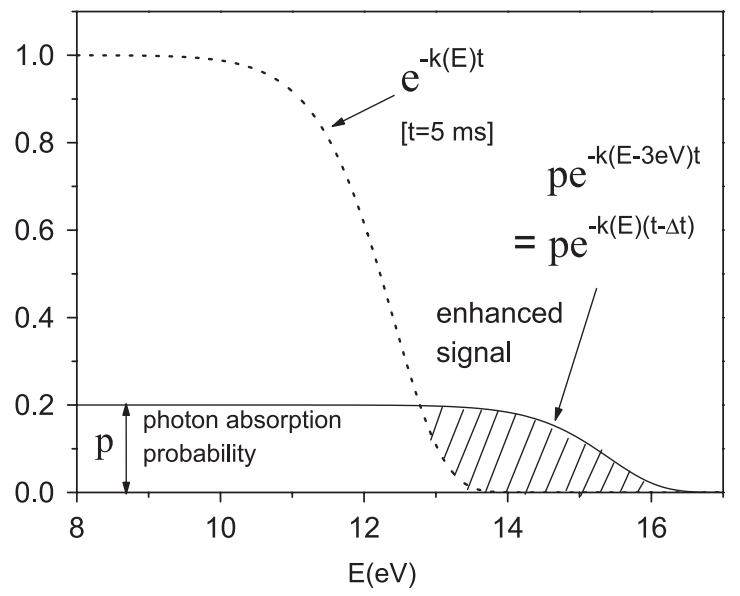

FIG. 2. Theoretical energy distributions right before and after one photon absorption. The dotted line is the energy distribution at $t=5 \mathrm{~ms}$ after creation, normalized to unity at low energy. The enhancement of the signal relative to the no-laser signal originates from the hatched area of the $p=20 \%$ of the ions that have absorbed one $3 \mathrm{eV}$ photon. 


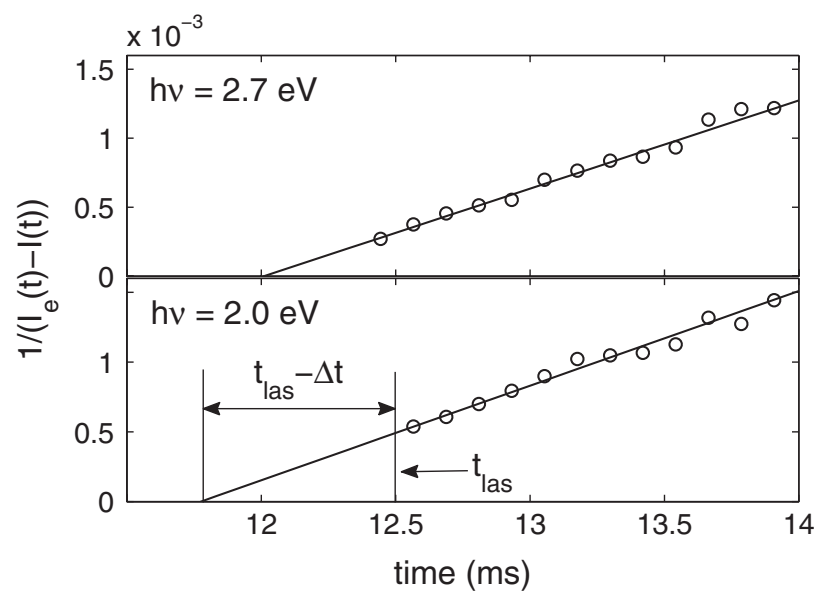

FIG. 3. Examples of extrapolation to zero reciprocal intensity, which gives the zero time of the laser enhanced signal, up to the minor corrections mentioned in the text.

the values of these parameters need not be known to perform the analysis. When only depletion occurs, the measured neutral signal is proportional to the time derivative of $E_{m}$

$$
I(t)=c \frac{1}{\ln (\omega t)^{2} t} \quad \text { (nonradiative), }
$$

where $c$ is a constant accounting for the density of molecules and instrumental factors. When the radiative cooling varies more slowly with excitation energy than electron emission, as is the case here with Planck-type radiation, the radiative cooling does not cause any significant distortion of the initial energy distribution. Then, in the presence of

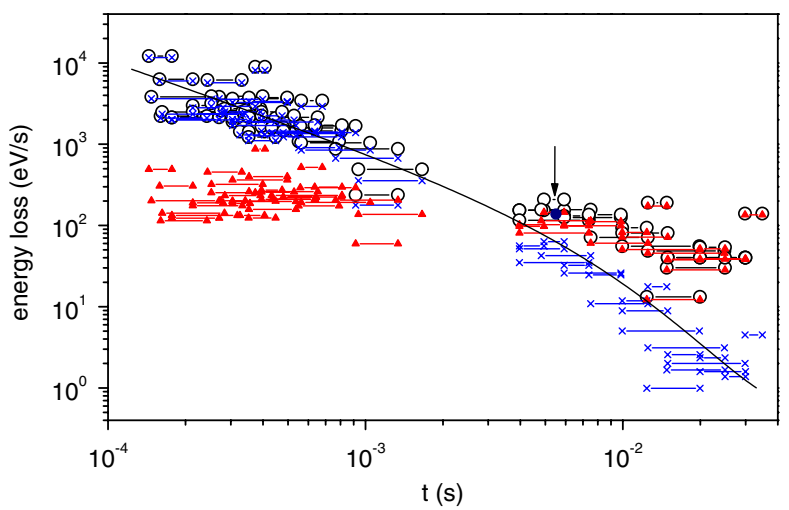

FIG. 4 (color online). Measured absolute cooling rates of $\mathrm{C}_{60}^{-}$. The ends of the time intervals are indicated with symbols. Most points are bunched from several close-lying points. Open black circles are total cooling rates. The red triangles are the radiative cooling component calculated by Eq. (9), and blue crosses the depletion cooling rate from Eq. (10). The line is the depletion cooling rate calculated with Eq. (3), fixing the single constant of proportionality required with the short time data. The arrow points to the $5.5 \mathrm{~ms}, 138 \mathrm{eV} / \mathrm{s}$ radiative cooling point modeled from the spontaneous decay curve in [18]. radiative cooling, the decay rate can be used to define an effective time, $t^{\prime}(t)$, which is the time where the rate would have the value observed in the absence of radiative cooling,

$$
I(t)=c \frac{1}{\ln \left(\omega t^{\prime}\right)^{2} t^{\prime}} \quad \text { (radiation present), }
$$

with the same constant $c$ as in Eq. (2). Alternatively this can be written as $I_{R}(t)=I_{N R}\left(t^{\prime}\right)$, where subscripts $R$ and $N R$ refer to the radiative and the hypothetical nonradiative case, respectively. With the experimentally observed time dependence of the decay rate the dependence of $t^{\prime}$ on $t$ can be found from this definition, fixing the constants by requiring that $t^{\prime} \rightarrow t$ for short times where radiative cooling is negligible. The expression is, to leading order in $1 / \ln (\omega t)$ and with the same $a_{1}, a_{2}$ as above,

$$
t^{\prime}=t \exp \left(-a_{1} t-a_{2} t^{2}\right),
$$

which is greater than $t$.

We disentangle the radiative cooling from the depletion as follows: The rate of decrease in the maximal energy in the ensemble is

$$
-\left.\frac{d E_{m}}{d t}\right|_{\text {tot }}=\frac{\Phi C_{v} / k_{B}}{\ln \left(\omega t^{\prime}\right)^{2} t^{\prime}} \frac{d t^{\prime}}{d t},
$$

with the radiative part

$$
-\left.\frac{d E_{m}}{d t}\right|_{\mathrm{rad}}=\frac{\Phi C_{v} / k_{B}}{\ln \left(\omega t^{\prime}\right)^{2} t^{\prime}}\left(\frac{d t^{\prime}}{d t}-1\right) .
$$

This assumes that radiation can be described as a continuous process, or that typical energies of emitted photons $\langle h \nu\rangle$ do not exceed the width of the energy region that contribute to the signal at any given time. For a $T^{6}$ dependence for the energy emission rate [15], this is well fulfilled. For comparison, an analysis of the laser induced decay of $\mathrm{Al}_{4}^{-}$gives a radiation intensity proportional to $T^{3.5}$ [16].

The integrated radiative energy loss is

$$
\begin{aligned}
-E_{\mathrm{rad}}\left(t_{1} ; t_{2}\right) & =\left.\int_{t_{1}}^{t_{2}} \frac{d E_{m}}{d t}\right|_{\mathrm{rad}} d t \\
& =h \nu-\int_{t_{1}}^{t_{2}} \frac{\Phi C_{v} / k_{B}}{\ln \left(\omega t^{\prime}\right)^{2} t^{\prime}} d t .
\end{aligned}
$$

The first term is simply the integral of the total energy loss. The term $h \nu$ may here refer to the energy of the absorbed photon or the difference between two photon energies if differences are taken according to the procedure described above. We can substitute the unknown factors in the integral of Eq. (7) if we note that

$$
h \nu=\frac{\Phi C_{v} / k_{B}}{\ln \left(\omega t_{1}^{\prime}\right)}-\frac{\Phi C_{v} / k_{B}}{\ln \left(\omega t_{2}^{\prime}\right)}=\frac{\Phi C_{v} / k_{B}}{\ln \left(\omega t_{1}^{\prime}\right) \ln \left(\omega t_{2}^{\prime}\right)} \ln \left(\frac{t_{2}^{\prime}}{t_{1}^{\prime}}\right) .
$$

Combined with Eq. (7) and approximating $\ln \left(\omega t^{\prime}\right)$ with a constant, we have 


$$
-E_{\mathrm{rad}}\left(t_{1} ; t_{2}\right) \simeq h \nu\left(1-\frac{1}{\ln \left(t_{2}^{\prime} / t_{1}^{\prime}\right)} \int_{t_{1}}^{t_{2}} \frac{1}{t^{\prime}} d t\right) .
$$

The cooling rates calculated with this formula are shown in Fig. 4. The radiative cooling rate varies only weakly between $130 \mu$ s to $30 \mathrm{~ms}$, but the relative importance of the radiative cooling changes dramatically during this time, from ca. $10 \%$ initially to above $95 \%$ of the cooling at latest times. The loss of energy from depletion alone is calculated correspondingly as

$$
-E_{\mathrm{dep}}\left(t_{1} ; t_{2}\right) \approx \frac{h \nu}{\ln \left(t_{2}^{\prime} / t_{1}^{\prime}\right)} \int_{t_{1}}^{t_{2}} \frac{1}{t^{\prime}} d t
$$

This shows the expected very strong time dependence, from almost all depletion cooling at submillisecond times to a few percent of the total, in a reduction by 3 orders of magnitude of the absolute signal.

Decay parameters that can be extracted from the data compare well with expected values. For $t=0.4 \mathrm{~ms}$, the (nonradiative) decay gives a value of $0.9 \mathrm{eV}$ for $\Phi C_{v} / k_{B} / \ln (\omega \times 0.4 \mathrm{~ms})^{2}$, with uncertainties estimated to be a factor 1.5 , which should be compared with the expected $0.98 \mathrm{eV}$ [17]. Figure 4 also shows the rate of depletion cooling calculated from the experimental value $\Phi C_{v} / k_{B} / \ln (\omega \times 0.4 \mathrm{~ms})^{2}=0.9 \mathrm{eV}$ and the expected dependence in Eq. (3) with $\omega=3 \times 10^{12} \mathrm{~s}^{-1}$, i.e., the expression

$$
-E_{\mathrm{dep}}=\frac{0.9 \mathrm{eV} \ln (\omega \times 0.4 \mathrm{~ms})^{2}}{\ln \left(\omega t^{\prime}\right)^{2} t^{\prime}} .
$$

The agreement between the calculated curve and the experimental data validates the assumptions leading to the Eqs. (9) and (10).

The time of crossover from depletion cooling to radiative cooling is given by the so-called radiation time $\tau$ [15], which here is given in terms of the radiated power $\dot{E}_{\text {rad }}$ by $\tau=C_{v} \Phi / k_{B} / \ln (\omega \tau)^{2} / \dot{E}_{\mathrm{rad}}(\tau)=7-8 \mathrm{~ms}$. This should be compared with the $5.5 \mathrm{~ms}$ value for $T=1356 \mathrm{~K}$ found in [18]. The difference is due almost exclusively to the smaller experimentally observed radiative cooling. For comparison, the value of the fit parameter used to fit the spontaneous decay curve here is $1 /\left(-a_{1}\right)=8.2 \mathrm{~ms}$.

In summary, we have measured the absolute cooling rate over a time range from $130 \mu$ s to $30 \mathrm{~ms}$ of fullerene anions $\mathrm{C}_{60}^{-}$produced in hot sources, in a proof-of-principles experiment. The cooling rates are the highest possible ones that can be observed for this ion for spontaneous cooling, and depend strongly on the time elapsed since production of the hot ion. The branching between energy loss by thermionic emission and the thermal radiation was measured and found to be consistent with previous modeling of spontaneous decay curves. The method, which is based on single photon excitation and the nonexponential decay of cluster ensembles, is generally applicable to ions of either charge state as long as the radiative cooling times are longer than the instrumental time scale of typically hundred $\mu \mathrm{s}$, provided the ions have a nonvanishing photoabsorption cross section.

The work was supported by the University of Gothenburg Nanoparticle Platform, STINT, the Swedish Research Council (VR), and in part by a Grant-in-Aid for Scientific Research (No. 19350015 and No. 20750015) from Japan Society for the Promotion of Science.

[1] M. Schmidt, R. Kusche, W. Kronmüller, B. von Issendorff, and H. Haberland, Phys. Rev. Lett. 79, 99 (1997).

[2] K. Hansen, A. Herlert, L. Schweikhard, and M. Vogel, Phys. Rev. A 73, 063202 (2006).

[3] G. A. Breaux, C. M. Neal, B. Cao, and M. F. Jarrold, Phys. Rev. Lett. 94, 173401 (2005).

[4] H. A. Michelsen, J. Chem. Phys. 118, 7012 (2003).

[5] L. Qi, H. F. Zhang, Z. Q. Hu, and P. K. Liaw, Phys. Lett. A 327, 506 (2004).

[6] P. Koskinen, H. Häkkinen, B. Huber, B. von Issendorff, and M. Moseler, Phys. Rev. Lett. 98, 015701 (2007).

[7] M. F. Jarrold, B. Cao, A. K. Starace, C. M. Neal, and O. Judd, J. Chem. Phys. 129, 014503 (2008).

[8] L. J. Allamandola, A. G. G. M. Tielens, and J. R. Barker, Astrophys. J. Suppl. Ser. 71, 733 (1989).

[9] A. G. G. M. Tielens, Annu. Rev. Astron. Astrophys. 46, 289 (2008).

[10] R. Visser, V. C. Geers, C.P. Dullemond, J. C. Augereau, K. M. Pontoppidan, and E. F. van Dishoeck, Astron. Astrophys. 466, 229 (2007).

[11] K. Hansen, J. U. Andersen, P. Hvelplund, S. P. Møller, U. V. Pedersen, and V. V. Petrunin, Phys. Rev. Lett. 87, 123401 (2001).

[12] S. Jinno, T. Takao, Y. Omata, A. Satou, H. Tanuma, T. Azuma, H. Shiromaru, K. Okuno, N. Kobayashi, and I. Watanabe, Nucl. Instrum. Methods Phys. Res., Sect. A 532, 477 (2004).

[13] S. P. Møller, Nucl. Instrum. Methods Phys. Res., Sect. A 394, 281 (1997).

[14] K. Hansen, J. U. Andersen, H. Cederquist, C. Gottrup, P. Hvelplund, M. O. Larsson, V. V. Petrunin, and H. T. Schmidt, Eur. Phys. J. D 9, 351 (1999).

[15] J. U. Andersen, C. Brink, P. Hvelplund, M. O. Larsson, B. Bech Nielsen, and H. Shen, Phys. Rev. Lett. 77, 3991 (1996).

[16] Y. Toker, O. Aviv, M. Eritt, M. L. Rappaport, O. Heber, D. Schwalm, and D. Zajfman, Phys. Rev. A 76, 053201 (2007).

[17] J. U. Andersen, C. Gottrup, K. Hansen, P. Hvelplund, and M. O. Larsson, Eur. Phys. J. D 17, 189 (2001).

[18] J. U. Andersen, P. Hvelplund, S. B. Nielsen, U. V. Pedersen, and S. Tomita, Phys. Rev. A 65, 053202 (2002). 